\title{
ADHD Medication in the Longer Term
}

\author{
Eric Taylor \\ Institute of Psychiatry, Psychology and NeuroScience, King’s College London, UK
}

\begin{abstract}
This review starts from the finding that there is strong persistence of ADHD problems into adult life, despite the availability of effective treatment with stimulants and other drugs. Short-term trials in adults reveal that those drugs can still be efficacious in adults. Pharmacoepidemiology studies, on the other hand, clearly show that most young people discontinue medication during adolescence. Neuropharmacology research suggests a waning of stimulant effects after a few years. Longitudinal studies are scarce but provide some evidence that prognosis is often determined by factors other than the core symptoms of inattentiveness and impulsive overactivity. The implications for clinical practice are discussed. Continuing care, especially during the follow-up after initial therapy, needs further development.
\end{abstract}

Keywords: ADHD, persistence of symptoms/problems, medication in the longer term, clinical prognosis, implications for clinical practice

\section{Introduction}

ADHD is a disability that often persists into adulthood. Inattentiveness, impulsiveness, and overactivity are the defining characteristics. Even unreferred cases in the community and those with high levels of the behavior problems not meeting the diagnosis criteria can continue to show impaired social development. Impulsiveness declines in absolute terms but remains deviant relative to age-matched peers (Taylor et al., 1996).

The effects in childhood include lack of friends, poor performance in school, and few constructive leisure activities. In adolescence, motor accidents become a considerable risk, and there may be academic underachievement (Faraone et al., 2006). Aggressive and antisocial behaviors and delinquency can complicate impulsiveness and overactivity (but not so much inattentiveness alone). Substance misuse is more common in those with ADHD than in the general population. Emotional dysregulation is very common and leads to later relationship problems and mood disorders (Stringaris et al., 2009).

Some children do escape the developmental problems or learn to live with them. Nevertheless, a systematic review by Shaw et al. (2012) found evidence for a wide range of adverse outcomes being more common in ADHD than in unaffected people over the lifespan. That review also suggested that those adverse outcomes are less common in those who have been treated than for untreated people with ADHD. This is encouraging, but the persistence of problems in the face of effective treatment remains a challenge to clinicians and researchers. This perspective article seeks to identify some potential limitations of treatment in the long term and to indicate how they might be overcome. Table 1 summarizes some of the possible limitations, not all of which may apply to everyone, and the potential implications for clinical practice that would arise if they did apply.

\section{Effect of Medication in the Long Term}

The picture summarized above of persisting disability for many is disappointing, not the least because a very effective therapy is available and widely used. The beneficial effects of psychostimulants over the short term have been clear since the 1930s (Taylor, 2009). The effects are large, with effect sizes relative to placebo around 0.9 SD (Faraone \& Buitelaar, 2010; NICE, 2009; SIGN, 2009). Few treatments in psychiatry can command the same level of positive evidence. The long-term effects do not seem so well established. Randomized controlled trials for periods of more than 12 weeks exist, but none has addressed the full developmental period from childhood through adolescence (Maia et al., 2017). Long-term randomized trials are not only difficult and expensive to carry out, they bring ethical problems of consent.

The Multimodal Treatment Study of ADHD (MTA), however, enabled a long-term and sufficiently large followup of a 14-month randomized comparison of children with ADHD who had been variously treated. Some had received 
Table 1. Possible constraints on the long-term value of medications and their implications

\begin{tabular}{ll}
\hline Potential Limitation & Clinical Implication \\
\hline Stimulants might be efficacious only in children, not adults. & Other therapies for adults \\
Poor compliance with medication might limit effectiveness. & Enhance motivation/adherence \\
The main prognostic factors might not be susceptible. & Extend range of interventions \\
Self-selection for favored therapy might lead to equivalence. & Advice and monitoring \\
Suboptimal service delivery re dosage and monitoring. & Enhance continuing reviews \\
The effect of medication might wear off with time. & Consider drug holidays \\
\hline
\end{tabular}

intensive behavioral management and psychological help, whereas others had received a careful regime of closely monitored medication, and yet another group had the benefit of both. Others again received only the usual treatment (whether medical or psychological) provided in their community. Over the period of 14 months that constituted the trial, there was an unequivocally better outcome for those who had received the "carefully crafted" medication.

The MTA investigators, however, followed up the treated children in an exemplary fashion. At the 2-year follow-up, the benefits persisted but at 3 years had faded, and at the 8- and 16-year follows-up there was no discernable advantage for any of the groups that had received the intensive therapies (Molina et al., 2009; Swanson et al., 2017). Their symptomatic outcomes and social adjustment were no better than those for the group that had received treatment as usual. This result must be seen in the context that, after the 14-month point, the groups were no longer as initially randomized. Indeed, only $53.5 \%$ of the participants providing saliva were shown to be adherent at all four measurement time points (Pappadopulos et al., 2009). Furthermore, even for those who remained in the condition of original assignment, the high level of fidelity to the detailed protocol of therapy would not have continued. The follow-up was therefore observational rather than experimental; but it is the best evidence of its kind available.

\section{What Might Be Responsible for Any Long-Term Limitation of Therapy?}

There are several possible reasons that demand separate consideration as they carry distinct implications for longterm clinical management.

\section{Medication is Not Effective in Adult Life?}

This is probably not the explanation. There have now been enough trials of treatment in adults to be confident that medication is still effective, and that cognitively based therapies and learning coping skills are promising. The Canadian Agency for Drugs and Technologies in Health (2011) provided a systematic review, and Cortese et al.
(2018) provided a network meta-analysis comparing therapies. Indeed, there is some evidence that adults with continuing symptoms make a better adjustment if their ADHD is being controlled with medication. Lichtenstein et al. (2012), for instance, found less offending behavior during periods of medication than in periods of abstinence. While this demonstrates effectiveness in the short to medium term in adult life, this is not the same as demonstrating long-term effectiveness. Some periods of abstinence may follow only short-term use; by definition some periods of use will represent recent commencement. Furthermore, deterioration during abstinence could reflect rebound rather than continuing value.

Similar considerations apply to findings such as those of Man et al. (2015) in young people up to the age of 19 in Hong Kong, namely, that the rate of trauma-related ED admission was lower during periods of exposure to medication than during nonexposed periods. This self-controlled series method, which relies on within-person comparisons, is a strong way of excluding many possible confounders, though it does not allow for consideration of possible fading of effect in long-term use.

\section{Medication Is Not Given or Taken?}

This does probably play a part in the persistence of symptoms and subsequent disability. The use of medication falls off quickly as young people get older. In a national (UK) survey by McCarthy et al. (2009), the key age for discontinuing was 16 years, which is also the age at which compulsory schooling ends. The reasons vary and include the presence of adverse effects, the adolescent desire for autonomy, stigma and hostile cultural attitudes to medication, simple forgetfulness, and the development of a despondent or nihilistic attitude toward the condition and its treatment. To overcome these obstacles, clinicians need to create a climate of communication that allows understanding of potential obstacles in the individual case. Taylor (2015) provided detailed implications for enhancing adherence.

This explanation, however, cannot be the whole story. In the MTA study cited above, the equifinality of outcomes for all therapies applied even to those who had consistent- 
ly taken medication throughout the follow-up (Swanson et al., 2017). The reasons for stopping medication may also include the fact that it is no longer effective.

The implication for clinical management is that followup should be careful and include both the symptomatic management of adverse effects (Graham et al., 2011) and the responsive discussion of attitudes, beliefs and the experience of taking medication (Ferrin \& Taylor, 2011; Singh et al., 2005).

\section{Medication Does Not Address the Main Prognostic Factors?}

The problems of people with ADHD are greater than the defining criteria. The core features of hyperactivity, inattention, and impulsivity are not necessarily the main characteristics of later disability. Comorbidity and complications are rife; stigma is pervasive; the reactions of family members, teachers, and peers can become negative. Associated aspects such as emotional dysregulation or aggressive conduct might be just as important as the level of core symptoms in driving adverse outcomes (Taylor 2009).

Nevertheless, most trials use the core DSM5 features as the outcome - and most interventions target the core features. This approach may either underestimate or overestimate the long-term impact of medication on personal and social functioning and the quality of life.

Nevertheless, (1) reducing these core features is promoted by guidelines as a means of improving longer-term outcomes; (2) those persons whose core symptoms persist are also those with the greater levels of adult occupational and functional disability (Klein et al., 2012). Clinical services should not rely solely on medication but address the aspects of each case that are likely to be detrimental in the long term.

\section{Self-Selection Implies That Most People Receive the Therapy That Best Suits Them?}

At the conclusion of the 14-month trial in the MTA, the original randomized assignment to treatments no longer applied. Those who perceived benefits from psychological treatment may have chosen to continue it, as did those who liked medication. The prediction would then indeed be that, if you get good advice and can follow it, you should end up with the therapy that suits you best - and have correspondingly similar results. The clinical implication would be to provide continued monitoring and advice as well as trials of different modes of intervention. Administrative constraints should not be allowed to prevent young people from having access to a range of treatments, or to prevent the young people and those responsible for them from sharing in the decisions about treatments and their sequencing.

\section{Continued Benefit of Interventions Might Require Very Careful Control of Therapies?}

After the 14-month trial in the MTA, the careful crafting of both behavioral and pharmacological interventions no longer applied. Perhaps if they had been carefully controlled and fully implemented, they would have produced some long-term benefit (Coghill \& Seth, 2015).

The clinical implication of this explanation for loss of effectiveness over time would then be clear, but dauntingly expensive. Furthermore, a key aspect of the intensive monitoring of the MTA medication was the progressive increase of dose. Perhaps this was connected to the decrement of growth in height and weight, a feature of the longterm outcome of the medicated group (Swanson et al., 2007, 2017).

\section{The Effect of Medication Wears Off with Time?}

Tolerance to psychostimulants is an expected feature of their pharmacology. Clinical experience suggests that the dose often needs to rise over the years to maintain effectiveness. Neuroimaging of the levels of synaptic dopamine indicate that the increase brought about by single doses of a stimulant is considerably less after a year of medication than it was when the same subjects were drugnaïve (Volkow et al., 2012). High dopamine transporter concentrations in the striatum - initially thought to be a diagnostic marker for ADHD - have been associated with long-term medication rather than with the disorder itself (Fusar-Poli et al., 2012). This could be one of the mechanisms promoting tolerance.

If tolerance is indeed significant for some treated people, then our attention should shift in research to understanding the mechanisms. In the meantime, it would be logical for clinical practice to include "holidays" from the drugs to allow the brain to recover its responsiveness.

\section{Clinical Recommendations}

In the absence of definitive evidence about the recommended length of pharmacotherapy, we recommend an individualized approach. Periodic periods off medication (e.g., 2 weeks every 2 years) should make it clear whether a drug is still desirable. (There should still be an option for returning to medication subsequently, if necessary.) Patients should become increasingly knowledgeable about the effects and potential adverse events of medication through their adolescence. Most do so and become intelligent consumers (Singh et al., 2005). Existential questions are raised by the short-term power of the therapies, and patients are often taxed to understand whether their 'real self' is that with ADHD or what appears when ADHD is 
controlled. The consequences include decision-making about whether to take the medication at all - or only when environmental demands for attention and self-control are high. A responsive discussion with a knowledgeable professional is greatly valued by most people with ADHD who share with other forms of disability, who grapple with the need to make a balance between overcoming its continuing problems and living within the limitations it imposes.

\section{Cultural Attitudes}

Independent of the scientific considerations above, social values play an important part in the decisions about whether medicines should be offered and, if offered, should be accepted in the longer term. International comparisons could be useful in generating ideas about best practice in who to treat and for how long (Beau-Lejdstrom \& Zito, 2018). Taylor (2017) used differences between administrative and research prevalence in different countries to suggest that both overdiagnosis and underrecognition of problems characterize different regions. Comparative data on longer-term outcomes might be useful in similar ways.

Public attitudes are influenced by education, mass media and social media, religious beliefs, and political ideologies. Attitudes toward therapies are often influenced by perceptions of whether they are natural or artificial. There is a view that "psychological problems should not be treated physically" - to which I would respond personally that the cause of a problem does not necessarily dictate how it should be managed. The question is rather what works. Medicalization may have a bad press but that does not make it wrong.

\section{What's New?}

The long-term outcome of ADHD is poor despite good drug treatments. Several explanations are suggested for the apparent waning of drug effectiveness. The development of pharmacological tolerance could be part of the story.

\section{Conclusion}

Researchers and clinicians should recognize the waning of drug effectiveness over time and seek methods of overcoming it.

This article is based on a talk delivered in Mannheim on the occasion of Professor Dittman's retirement.

\section{References}

Beau-Lejdstrom, R., \& Zito, J.M. (2018). Getting to long-term effectiveness and safety of attention-deficit hyperactivity disorder medications. Clinical Pharmacology and Therapeutics, 104, 610-612.

Canadian Agency for Drugs and Technologies in Health. (2011). Pharmacological and non-pharmacological therapies for adults with attention-deficit/hyperactivity disorder: Systematic review and meta-analysis of clinical evidence. Health Technology Assessment Database. http://www.crd.york.ac.uk/crd web/ShowRecord.asp?LinkFrom=OAI\&ID=32011001514

Coghill, D., \& Seth, S. (2015). Effective management of attentiondeficit/hyperactivity disorder (ADHD) through structured reassessment: The Dundee ADHD Clinical Care Pathway. Child and Adolescent Psychiatry and Mental Health, 19(1), 52-65.

Cortese, S., Adamo, N., Del Giovane, C., Mohr-Jensen, C., Hayes, A.J., Carucci, S., ... \& Hollis, C. (2018). Comparative efficacy and tolerability of medications for attention-deficit hyperactivity disorder in children, adolescents, and adults: A systematic review and network meta-analysis. The Lancet Psychiatry, 5, 727-738.

Faraone, S.V., Biederman, J., \& Mick, E. (2006). The age-dependent decline of attention deficit hyperactivity disorder: A meta-analysis of follow-up studies. Psychological Medicine, 36, 159-165.

Faraone, S.V., \& Buitelaar, J. (2010). Comparing the efficacy of stimulants for ADHD in children and adolescents using metaanalysis. European Child and Adolescent Psychiatry, 19, 353-364.

Ferrin, M., \& Taylor, E. (2011). Child and caregiver issues in the treatment of attention deficit-hyperactivity disorder: Education, adherence and treatment choice. Future Neurology, 6, 399-413.

Graham, J., Banaschewski, T., Buitelaar, J., Coghill, D., Danckaerts, M., Dittmann, R. W., ... \& Hulpke-Wette, M. (2011). European guidelines on managing adverse effects of medication for ADHD. European Child and Adolescent Psychiatry, 20, 17-37.

Fusar-Poli, P., Rubia, K., Rossi, G., Sartori, G., \& Balottin, U. (2012). Striatal dopamine transporter alterations in ADHD: Pathophysiology or adaptation to psychostimulants? A meta-analysis. American Journal of Psychiatry, 169, 264-272.

Klein, R.G., Mannuzza, S., Olazagasti, M.A.R., Roizen, E., Hutchison, J.A., Lashua, E.C., \& Castellanos, F.X. (2012). Clinical and functional outcome of childhood attention-deficit/hyperactivity disorder 33 years later. Archives of General Psychiatry, 69, $1295-1303$

Lichtenstein, P., Halldner, L., Zetterqvist, J., Sjölander, A., Serlachius, E., Fazel, S., ... \& Larsson, H. (2012). Medication for attention deficit-hyperactivity disorder and criminality. New England Journal of Medicine, 367, 2006-2014.

McCarthy, S., Asherson, P., Coghill, D., Hollis, C., Murray, M., Potts, L., ... \& Wong, I.C. (2009) Attention-deficit hyperactivity disorder: Treatment discontinuation in adolescents and young adults. British Journal of Psychiatry, 194, 273-277.

Maia, C.R.M., Cortese, S., Caye, A., Deakin, T.K., Polanczyk, G.V., Polanczyk, C.A., \& Rohde, L.A.P. (2017). Long-term efficacy of methylphenidate immediate-release for the treatment of childhood ADHD: A systematic review and meta-analysis. Journal of Attention Disorders, 21, 3-13.

Man, K.K., Chan, E.W., Coghill, D., Douglas, I., Ip, P., Leung, L.P., ... \& Wong, I.C. (2015). Methylphenidate and the risk of trauma. Pediatrics, $135,40-48$.

Molina, B. S., Hinshaw, S. P., Swanson, J. M., Arnold, L. E., Vitiello, B., Jensen, P.S. ... \& Elliott, G.R. (2009). The MTA at 8 years: Prospective follow-up of children treated for combined-type ADHD in a multisite study. Journal of the American Academy of Child and Adolescent Psychiatry, 48, 484-500. 
National Institute for Clinical Excellence. (2009). Attention deficit hyperactivity disorder. British Psychological Society and The Royal College of Psychiatrists Leicester \& London, retrieved from www.nice.org.uk/CG72

Pappadopulos, E., Jensen, P.S., Chait, A. R., Arnold, L.E., Swanson, J.M., Greenhill, L. L., ... \& Cooper, T. (2009). Medication adherence in the MTA: Saliva methylphenidate samples versus parent report and mediating effect of concomitant behavioral treatment. Journal of the American Academy of Child and Adolescent Psychiatry, 48, 501-510.

Shaw, M., Hodgkins, P., Caci, H., Young, S., Kahle, J., Woods, A. G., \& Arnold, L.E. (2012). A systematic review and analysis of longterm outcomes in ADHD: Effects of treatment and non-treatment. BMC Medicine, 10, 99-114. Retrieved from http://www. biomedcentral.com/content/pdf/1741-7015-10-99.pdf

Scottish Intercollegiate Guidelines Network. (2009). Management of attention deficit and hyperkinetic disorders in children and young people. SIGN, Edinburgh. Retrieved from www.sign.ac. uk/guidelines/fulltext/112.

Singh, I. (2005). Will the "real boy" please behave: Dosing dilemmas for parents of boys with ADHD. American Journal of Bioethics, 5 , 34-47.

Stringaris, A., Cohen, P., Pine, D. S., \& Leibenluft, E. (2009). Adult outcomes of youth irritability: A 20-year prospective community-based study. American Journal of Psychiatry, 166, $1048-1054$

Swanson, J.M., Elliott, G. R., Greenhill, L.L., Wigal, T., Arnold, L.E., Vitiello, B., ... \& Newcorn, J. H. (2007). Effects of stimulant medication on growth rates across 3 years in the MTA follow-up. Journal of the American Academy of Child and Adolescent Psychiatry, 46, 1015-1027.

Swanson, J. M.,Arnold, L. E., Molina, B.S., Sibley, M.H., Hechtman, L. T., Hinshaw, S. P., ... \& Nichols, Q. (2017), Young adult outcomes in the follow-up of the multimodal treatment study of attentiondeficit/hyperactivity disorder: Symptom persistence, source discrepancy, and height suppression. Journal of Child Psychology and Psychiatry, 58(6), 663-678. doi 10.1111/jcpp.12684

Taylor, E. (2009). Developing ADHD. Journal of Child Psychology and Psychiatry, 50, 126-132. (PMID: 19076263)
Taylor, E. (2015). Pharmacological, medically-led and related treatments. Rutter's Child and Adolescent Psychiatry, 6th edition, Wiley-Blackwell, 559-572.

Taylor, E. (2017). Attention deficit hyperactivity disorder: Overdiagnosed or diagnoses missed? Archives of Disease in Childhood, 102, 376-379.

Taylor, E., Chadwick, O., Heptinstall, E., \& Danckaerts, M. (1996). Hyperactivity and conduct problems as risk factors for adolescent development. Journal of the American Academy of Child \& Adolescent Psychiatry, 35, 1213-1226.

Volkow, N.D., Wang, G.J., Tomasi, D., Kollins, S.H., Wigal, T.L., Newcorn, J. H., ... \& Swanson, J. (2012) Methylphenidate-elicited dopamine increases in ventral striatum are associated with long-term symptom improvement in adults with attention deficit hyperactivity disorder. Journal of Neuroscience, 32, 841-949.

\section{Conflicts of Interests}

The author declares book royalties from Oxford University Press and Blackwell Wiley; Trustee for Autistica and Place2Be.

\section{History}

Manuscript submitted: 24.09.2018

Accepted after revision: 07.07.2019

Published online: 23.04.2019

\section{Eric Taylor}

FRCPsych, FMedSci

Institute of Psychiatry, Psychology and NeuroScience

King's College London

DeCrespigny Park, London SE5 8AF

United Kingdom

eric.taylor@kcl.ac.uk 